\title{
An Error Analysis for Numerical Multiple Integration. II
}

\author{
By Robert E. Barnhill*
}

1. Introduction. This paper is a continuation of earlier work [5] and it is concerned with bounding the error of cubatures for a certain class of analytic functions. This paper describes the application of the hypercircle inequality [12] to the problem of numerical multiple integration and the resulting optimal cubatures. A convergence theorem is given for the optimal cubatures, as well as numerical examples for some particular functions integrated over a square. The optimal cubatures can be compared with what the author has called minimum norm cubatures [5]. New results are given in this paper concerning asymptotic properties of the minimum norm cubatures and these, in turn, imply results concerning the optimal cubatures.

2. Derivation of the Optimal Cubatures. The hypercircle inequality was originally described by Synge [18] and reformulated by Golomb and Weinberger [12] and Davis [10]. It has been used in quadrature theory for analytic functions by Valentin [19] and the author [2] and for functions with integrable $n$th derivative by Secrest [15], [16]. In the latter cases, this method leads to the theory of spline approximation, which has a large literature to which references can be found in Secrest's articles and in the paper by Birkhoff and de Boor [7].

The cubatures derived from the hypercircle inequality are "optimal" in a certain sense, and hence this name. Suppose that we want to compute $L(f)=\iint f$, for some given function $f$, where the integral is over the square $0 \leqq x, u \leqq 1$, and that the following two types of information are available:

$$
f\left(z_{k}, w_{k}\right)=\alpha_{k}, \quad k=1, \cdots, n
$$

and

$$
\|f\| \leqq r \quad \text { for some } r>0,
$$

where the norm is defined below.

Without some restriction on the function space to which $f$ belongs, it need not be the case that (1) and (2) imply anything about $L(f)$. We consider certain analytic functions that are uniformly bounded in norm. We write the formulas for two dimensions and it will be clear how to extend them to $N$ dimensions. Let $E_{\rho}$ be the ellipse with foci at \pm 1 , semimajor axis $a$, semiminor axis $b=\left(a^{2}-1\right)^{1 / 2}$, and $\rho=(a+b)^{2}$. Then $E_{\rho} \times E_{\rho^{\prime}}$ is $\left\{(z, w): z\right.$ is in $E_{\rho}$ and $w$ is in $\left.E_{\rho^{\prime}}\right\}$. To simplify the writing, we let $\rho=\rho^{\prime}$, but it will be clear how to alter the formulas for the more general case. Let $L^{2}\left(E_{\rho} \times E_{\rho}\right)$ be the set of functions that are analytic inside $E_{\rho} \times E_{\rho}$ and such that

Received May 19, 1967.

* This work was performed while the author was on leave at Brown University, Division of Applied Mathematics, Providence, Rhode Island. 


$$
\|f\|^{2}=\iiint \int_{E_{\rho} \times E_{\rho}}|f(z, w)|^{2} d x d y d u d v
$$

exists, where $z=x+i y, w=u+i v, x, y, u$ and $v$ are real, and the integral is over the region enclosed by $E_{\rho} \times E_{\rho}$. (The four-dimensional real integral above is defined to be $\|f\|^{2}$.) For simplicity, we denote $L^{2}\left(E_{\rho} \times E_{\rho}\right)$ by $L^{2}$. For the given function $f$, we assume that $\|f\| \leqq r$ and $f\left(z_{k}, w_{k}\right)=\alpha_{k}$, where the $\left(z_{k}, w_{k}\right)$ are in $E_{\rho} \times E_{\rho}$. We define the closed hyperdisc $C_{n}$ as $\left\{g\right.$ in $L^{2}:\|g\| \leqq r$ and $\left.g\left(z_{k}, w_{k}\right)=\alpha_{k}, \quad k=1, \cdots, n\right\}$. Let $U$ denote $\left\{g\right.$ in $L^{2}:\|g\| \leqq r$ and $\left.g\left(z_{k}, w_{k}\right)=0, k=1, \cdots, n\right\}$. Then the hypercircle inequality can be stated as follows

$$
|L(f)-L(u)| \leqq\|L\|_{U}\left(r^{2}-\|u\|^{2}\right)^{1 / 2},
$$

where $u$ is the element of least norm in $C_{n}$. $u$ has the property that $L(u)$ can be considered as the cubature sum (see Eq. (4)), so that the left-hand side of (3) is the error made in integrating $f$. This bound on the error is optimal in the sense that it holds for all functions $f$ satisfying (1) and (2), and is an equality for at least one $\operatorname{such} f$.

The space $L^{2}$ is a Hilbert space, with the inner product

$$
(f, g)=\iiint \int f(z, w) \overline{g(z, w)} d x d y d u d v \text {. }
$$

A complete orthonormal sequence is known [5] for $L^{2}$ and it is the following: $\left\{p_{r}(z) p_{s}(w)\right\}_{r, s=0}^{\infty}$, where $p_{r}(z)=2\left\{(r+1) /\left[\pi\left(\rho^{r+1}-\rho^{-r-1}\right)\right]\right\}^{1 / 2} \cdot U_{r}(z)$, $p_{s}(w)=2\left\{(s+1) /\left[\pi\left(\rho^{s+1}-\rho^{-s-1}\right)\right]\right\}^{1 / 2} U_{s}(w)$ and $U_{r}(z)$ is the $r$ th Tchebycheff polynomial of the second kind. In $L^{2}$, the functional $L$ and the point functionals $L_{k}(f)=f\left(z_{k}, w_{k}\right)$ are bounded and hence have representers, by the Riesz representation theorem. Specifically, $L_{k}(f)=\left(f, h_{k}\right)$, where

$$
\begin{aligned}
h_{k}(z, w) & =\sum_{r, s=0}^{\infty} p_{r}(z) p_{s}(w) \overline{p_{r}\left(z_{k}\right)} \overline{p_{s}\left(w_{k}\right)} \\
& =\sum_{r, s} \alpha(r, \rho) \alpha(s, \rho) U_{r}(z) U_{s}(w) \overline{U_{r}\left(z_{k}\right)} \overline{U_{s}\left(w_{k}\right)}
\end{aligned}
$$

where $\alpha(r, \rho)=4(r+1) /\left[\pi\left(\rho^{r+1}-\rho^{-r-1}\right)\right]$. Also, $L(f)=(f, h)$, where

$$
h(z, w)=\sum_{r, s} \alpha(r, \rho) \alpha(s, \rho) \beta(r) \beta(s) U_{r}(z) U_{s}(w),
$$

and $\beta(r)=\left[1+(-1)^{r}\right] /(r+1)$. The formulas given by Golomb and Weinberger can now be applied, the results being the following:

$$
\begin{aligned}
L(u) & =\sum_{i, j=1}^{n} \Phi_{i j}^{-1} \alpha_{j}\left(h_{i}, h\right), \\
(u, u) & =\sum_{i, j} \Phi_{i j}^{-1} \alpha_{j} \alpha_{i}, \\
\|L\|_{U} & =\left\{(h, h)-\sum_{i, j} \Phi_{i j}^{-1}\left(h, h_{i}\right)\left(h, h_{j}\right)\right\}^{1 / 2},
\end{aligned}
$$

where $\Phi$ is the matrix $\left(\left(h_{i}, h_{j}\right)\right)$ with the inverse matrix $\Phi^{-1}=\left(\Phi_{i j}^{-1}\right)$. The necessary inner products are the following: 


$$
\begin{aligned}
\left(h_{i}, h_{j}\right) & =\sum_{r, s} \alpha(r, \rho) \alpha(s, \rho) U_{r}\left(z_{i}\right) \overline{U_{r}\left(z_{j}\right)} U_{s}\left(w_{i}\right) \overline{U_{s}\left(w_{j}\right)} \\
\left(h, h_{i}\right) & =\sum_{r, s} \alpha(r, \rho) \alpha(s, \rho) \beta(r) \beta(s) \overline{U_{r}\left(z_{i}\right)} \overline{U_{s}\left(w_{i}\right)} \\
(h, h) & =\sum_{r, s} \alpha(r, \rho) \alpha(s, \rho)\{\beta(r) \beta(s)\}^{2} .
\end{aligned}
$$

Also, from the expression for $L(u)$, we see that $L(u)=\sum_{j=1}^{n} A_{j} \alpha_{j}$, so that

$$
A_{j}=\sum_{i=1}^{n} \Phi_{i j}^{-1}\left(h_{i}, h\right), \quad j=1, \cdots, n .
$$

We remark that the integration functional $L$ can be taken over an arbitrary closed and bounded region in $L^{2}$, instead of over the square, and that the same formulas apply with the change that $\beta(r) \beta(s)$ is replaced by $\iint U_{r}(z) U_{s}(w)$.

3. Convergence of the Optimal Cubatures. Let $r_{n}$ be $\|L\|_{U(n)}\left\{r^{2}-\left\|u_{n}\right\|^{2}\right\}^{1 / 2}$, where we have indicated the dependence of the set $U$ and the optimal approximation $u$ on $n$. 'The convergence question is the following: Under what conditions does $r_{n} \rightarrow 0$ as $n \rightarrow \infty$ ? We remark that we have already made one nontrivial assumption, which is that our original function $f$ is in $C_{n}$, i.e., $C_{n}$ is not empty, $n=1, \cdots$. The author [4] has pointed out some sufficient conditions for this existence assumption in several one-dimensional cases. However, for more than one dimension, the corresponding complex variable results are apparently unknown. In this theorem we consider the complex point functionals $L_{k}(f)=f\left(z_{k}, w_{k}\right)$, with $z_{k}$ and $w_{k}$ complex.

Theorem 1. Let $X$ be a Hilbert space with $L_{1}, L_{2}, \cdots$ linearly independent elements in the dual space $X^{*}$. Let $f$ be a function such that $\|f\| \leqq r$ and $L_{k}(f)=\alpha_{k}$, $k=1, \cdots$. If the $L_{k}$ are complete in $X^{*}$, then $\lim r_{n}=0$.

The proof of this theorem is independent of the dimension and so is the same as that previously given for one dimension [4].

In one dimension, if the base points have an accumulation point inside $E_{\rho}$, then the point functionals are complete and $f$ is uniquely determined. In more than one dimension, an additional assumption must be made to guarantee the completeness of such point functionals.

Corollary 1.1 If the points $\left(z_{k}, w_{k}\right)$ have an accumulation point inside $E_{\rho} \times E_{\rho}$ and do not lie on any analytic hypersurface, then $\lim r_{n}=0$.

We remark that if the points $\left(z_{k}, w_{k}\right)$ are all real, then the $L_{k}$ are complete. This is the case of the most practical interest, of course.

It is interesting to note that Theorem 1 holds for any $L$ in $X^{*}$, so that the same theorem yields results on the convergence of optimal schemes for interpolation, numerical differentiation, etc. Theorem 1 also yields results concerning the uniform convergence of the minimum norm cubatures. We recall that a sequence of cubatures $Q_{n}$ converges uniformly means that $\left\|R_{n}\right\| \rightarrow 0$ as $n \rightarrow \infty$ [9].

CoRollary 1.2 If the point functionals $L_{k}$ are complete, then the corresponding minimum norm cubatures converge uniformly.

Proof. This corollary follows from the fact that $\|L\|_{U(n)} \rightarrow 0$ as $n \rightarrow \infty$ and $\|L\|_{U(n)}=\min _{A}\left\|R_{n}\right\|$.

From Corollary 1.2 follows 
Corollary 1.3 The sequence of cubatures which are of minimum norm with respect to the weights and nodes is uniformly convergent.

4. Order of Convergence of the Weights of the Minimum Norm Cubatures. In the paper that preceded this one, the following result was given: If we have an interpolatory cubature of $n$ points with precision $d$, where $n=C_{d+2,2}$, and the cubature weights are denoted by $W_{i}, i=1, \cdots, n$, then the corresponding minimum norm weights $A_{i}$ have the property that $A_{i} \rightarrow W_{i}, i=1, \cdots, n$, as $\rho \rightarrow \infty$. The next theorem is a stronger result, which gives the order of this convergence, as $\rho \rightarrow \infty$. For a special case of this theorem, the reader is referred to Valentin [19]. We consider $m$-dimensional rules with the vectors of the cubature weights being $W_{k}$, the vectors of nodes being $\bar{z}_{k}, k=1, \cdots, n$.

THEOREM 2. Let the $W_{k}$ and $\bar{z}_{k}$ determine a cubature with precision $d$ and let the minimum norm weights determined by the $\bar{z}_{k}$ be denoted by $A_{k}$, with $\epsilon_{k}=A_{k}-W_{k}$. Then $\epsilon_{k}=O\left(\rho^{-(d+1)}\right), k=1, \cdots, n$.

Proof. We give the proof for two dimensions and a way to extend it to $m$ dimensions. For the two-dimensional case, we denote the vector $\bar{z}_{k}$ by $\left(z_{k}, w_{k}\right)$.

The normal equations that result in minimizing $\left\|R_{n}\right\|$ are the following

$$
\begin{gathered}
\sum_{k=1}^{n} A_{k} \sum_{r=0}^{\infty} \sum_{s=0}^{\infty}\left(\frac{r+1}{\rho^{r+1}-\rho^{-r-1}}\right)\left(\frac{s+1}{\rho^{s+1}-\rho^{-s-1}}\right) U_{r}\left(z_{k}\right) U_{s}\left(w_{k}\right) U_{r}\left(z_{l}\right) U_{s}\left(w_{l}\right) \\
=\sum_{r=0}^{\infty} \sum_{s=0}^{\infty} \beta(r) \beta(s)\left(\frac{r+1}{\rho^{r+1}-\rho^{-r-1}}\right)\left(\frac{s+1}{\rho^{s+1}-\rho^{-s-1}}\right) U_{r}\left(z_{l}\right) U_{s}\left(w_{l}\right), \\
\quad l=1, \cdots, n .
\end{gathered}
$$

We substitute $W_{k}+\epsilon_{k}$ for $A_{k}$ in the above equations and recall that the rule's having precision $d$ means that all polynomials in two variables of total degree not greater than $d$ are integrated exactly using the weights $W_{k}$ and nodes $\left(z_{k}, w_{k}\right)$. Hence we can show that

$$
\sum_{k} \epsilon_{k} \sum_{r, s}\left[(r+1)(s+1) /\left(\rho^{r+1}-\rho^{-r-1}\right)\left(\rho^{s+1}-\rho^{-s-1}\right)\right] U_{r}\left(z_{k}\right) U_{s}\left(w_{k}\right) U_{r}\left(z_{l}\right) U_{s}\left(w_{l}\right)
$$

is $O\left(\rho^{-(d+3)}\right)$, as $\rho \rightarrow \infty$. However, the coefficient matrix of the $\epsilon_{k}$ has elements of $O\left(\rho^{-2}\right)$, so that the $\epsilon_{k}$ are of $O\left(\rho^{-(d+1)}\right)$.

To extend the above proof to $m$ dimensions, we note that, in the above, $O\left(\rho^{-(d+1+2)}\right)$ is replaced by $O\left(\rho^{-(d+1+m)}\right)$ and $O\left(\rho^{-2}\right)$ by $O\left(\rho^{-m}\right)$, respectively, which yields the same conclusion. Q.E.D.

We state one corollary pertaining to one-dimensional rules:

CoRollary 2.1 If the $\bar{z}_{k}$ are given and the $W_{k}$ are the corresponding interpolatory quadrature weights, then $\epsilon_{k}$ is at least $O\left(\rho^{-n}\right)$. If the $\bar{z}_{k}$ are the Gaussian nodes, then $\epsilon_{k}=O\left(\rho^{-2 n}\right)$.

We note that this corollary can be substantiated numerically from, e.g., Table 1 in [1].

Remark. At the outset of Section 4, we remarked that Theorem 2 is a stronger result than the one given in the preceding paper. The earlier line of reasoning should not be neglected, however, for the author conjectures that it will lead to the following result: given the hypotheses of Stroud's Theorem 2 [17], then the weights and nodes of the minimum norm cubature converge to the corresponding weights 
and nodes obtained from the appropriate orthogonal polynomials in several variables. For completeness, we remark that Stroud's theorem states sufficient conditions for the common zeros of a set of orthogonal polynomials to be used as cubature nodes and for the cubature so formed to have precision properties analogous to the Gaussian quadratures in one dimension.

It was remarked in the Introduction that the asymptotic properties of the minimum norm cubatures imply results on the asymptotic properties of the optimal cubatures. These results follow from the fact that the optimal cubature weights are the same as the minimum norm cubature weights. This remark applies only to cubatures with given nodes, of course.

5. Numerical Examples. This section is concerned with the numerical application of the results given in Section 2. As remarked in Section 4, the weights of the optimal cubatures are the same as the weights of the minimum norm cubatures with the same nodes, and so these two types of cubature yield the same error. However, the bounds on the error are uniformly smaller for the optimal cubatures and it is of interest to compare the two bounds to each other as well as to the actual error. Two functions are considered on the square $-1 \leqq x, u \leqq 1$; namely, $f_{1}(x, u)=e^{x+u}$ and $f_{2}(x, u)=\cos x \cos u$. Four cubatures are considered and these are the following: the cross-product trapezoidal rule $T_{2} \times T_{2}$, the cross-product Gaussian rule $G_{2} \times G_{2}$, Lyness' nine-point rule [11, p. 141], and the cross-product Gaussian rule $G_{3} \times G_{3}$. Lyness' rule is formed by taking all points symmetric to the three generators listed in Table 3 . We remark that the fact that $\|L\|_{U}=\min _{A}\left\|R_{n}\right\|$ was used to simplify the calculations.

The definitions of the symbols in Tables 1-4 are as follows: $a$ is the semimajor axis of the ellipse $E_{\rho} ; E_{i}$ is the actual error made in integrating $f_{i} ; B_{i}$ is the error bound of the minimum norm cubature; $B_{i}{ }^{H}$ is the error bound of the optimal cubature, $i=1,2$. The numbers are expressed in floating-point notation, the sign and following two digits being the appropriate power of ten.

TABLE $1 . \quad T_{2} \times T_{2}$

\begin{tabular}{ccccccc}
\hline$a$ & $E_{1}$ & $E_{2}$ & $B_{1}$ & $B_{2}$ & $B_{1}{ }^{H}$ & $B_{2}{ }^{H}$ \\
\hline 1.2 & $4.60+00$ & $2.72+00$ & $4.18+01$ & $5.72+00$ & $4.16+01$ & $5.72+00$ \\
1.5 & $1.41+00$ & $2.33+00$ & $5.82+01$ & $8.31+00$ & $5.75+01$ & $8.28+00$ \\
2.0 & $2.01+00$ & $1.91+00$ & $9.46+01$ & $1.47+01$ & $9.41+01$ & $1.47+01$ \\
5.0 & $3.96+00$ & $1.67+00$ & $5.86+03$ & $1.20+03$ & $5.86+03$ & $1.20+03$ \\
\hline
\end{tabular}

TABLE $2 . \quad G_{2} \times G_{2}$

\begin{tabular}{ccccccc}
\hline$a$ & $E_{1}$ & $E_{2}$ & $B_{1}$ & $B_{2}$ & $B_{1}{ }^{H}$ & $B_{2}{ }^{H}$ \\
\hline 1.2 & $5.92-01$ & $3.08-01$ & $1.55+01$ & $2.11+00$ & $1.51+01$ & $1.76+00$ \\
1.5 & $7.81-02$ & $4.54-02$ & $7.62+00$ & $1.09+00$ & $7.54+00$ & $1.03+00$ \\
2.0 & $3.87-02$ & $2.52-02$ & $5.05+00$ & $7.86-01$ & $5.04+00$ & $7.82-01$ \\
5.0 & $3.62-02$ & $2.39-02$ & $4.13+01$ & $8.43+00$ & $4.13+01$ & $8.43+00$ \\
\hline
\end{tabular}


TABLE 3. Lyness Generators: $(0.632456,0.0),(1.0,1.0),(0.0,0.0)$

\begin{tabular}{ccccccc}
\hline$a$ & $E_{1}$ & $E_{2}$ & $B_{1}$ & $B_{2}$ & $B_{1}{ }^{H}$ & $B_{2}{ }^{H}$ \\
\hline 1.2 & $5.62-01$ & $1.08-02$ & $1.32+01$ & $1.81+00$ & $1.30+01$ & $1.29+00$ \\
1.5 & $2.91-02$ & $1.93-03$ & $3.79+00$ & $5.41-01$ & $3.74+00$ & $4.85-01$ \\
2.0 & $5.45-03$ & $6.64-03$ & $1.26+00$ & $1.95-01$ & $1.25+00$ & $1.89-01$ \\
5.0 & $8.32-03$ & $6.66-03$ & $1.46+00$ & $2.99-01$ & $1.46+00$ & $2.98-01$ \\
\hline
\end{tabular}

TABLE $4 . \quad G_{3} \times G_{3}$

\begin{tabular}{cccccccc}
\hline$a$ & $E_{1}$ & $E_{2}$ & $B_{1}$ & $B_{2}$ & $B_{1}{ }^{H}$ & $B_{2}{ }^{H}$ \\
\hline 1.2 & $7.76-02$ & $3.49-02$ & $5.58+00$ & $7.63-01$ & $5.44+00$ & $5.50-01$ \\
1.5 & $2.04-03$ & $2.14-04$ & $1.36+00$ & $1.93-01$ & $1.34+00$ & $1.73-01$ \\
2.0 & $3.53-04$ & $2.14-04$ & $4.42-01$ & $6.87-02$ & $4.39-01$ & $6.65-02$ \\
5.0 & $3.08-04$ & $2.07-04$ & $5.13-01$ & $1.05-01$ & $5.13-01$ & $1.05-01$ \\
\hline
\end{tabular}

6. Conclusions. For fixed nodes the error bound (3) given by the hypercircle inequality is the smallest possible. Thus, it is perhaps surprising that these error bounds are so little better than the error bounds of the minimum norm cubatures in the numerical examples of Section 5. We observe that both bounds are conservative in these examples, being on the order of $10^{2}$ to $10^{3}$ larger than the actual errors. However, there is a function in the hyperdisc $C$ for which the error bound is the actual error and so the bounds are not always conservative.

If we consider the cubature nodes as variable, then various improvements suggest themselves. Two of these are minimum norm cubatures with variable nodes and optimal cubatures with the error bound (3) minimized with respect to the nodes. The former possibility is currently under investigation and a variation of the latter, for one dimension, has been considered [3].

We conclude with a few remarks of interest to users of these cubature rules. For given nodes and integration over the square, formulas (3)-(7) yield the corresponding optimal cubature and error bound. As remarked in Section 2, optimal cubatures over other regions can be derived if $U_{r}(z) U_{s}(w)$ can be integrated over the region. If the products of the Tchebycheff polynomials cannot be integrated over the region of interest, then, assuming that the functions to be integrated are analytic in a sufficiently large region, we can use a different Hilbert space of functions which has the feature that products of the complex monomials $z^{r} w^{s}$ form a complete orthogonal system. This Hilbert space consists of functions analytic inside the cross-product of two circles and bounded in the $L^{2}$-norm over this crossproduct region. For more information on this space the reader is referred to [6], [8], [19].

Acknowledgements. The author is grateful to Philip J. Davis for setting up the year at Brown University during which this research was done. The author also acknowledges Steven L. Shrier for carrying out the computations.

This research was supported by the Office of Naval Research under Contract Nonr 562(36) to Brown University and by the National Science Foundation under 
Grant GP 5906 to the University of Utah. A part of the computing time was made available by the National Science Foundation under Grant GP 4825 to Brown University.

Division of Applied Mathematics

Brown University

Providence, Rhode Island 02912

Department of Mathematics

University of Utah

Salt Lake City, Utah 84112

1. R. E. Barnhill \& J. A. Wixom, "Quadratures with remainders of minimum norm. I," Math. Comp., v. 21, 1967, pp. 66-75.

2. R. E. BARNhILL, "Optimal quadratures in $L^{2}\left(E_{\rho}\right)$. I," SIAM J. Numer. Anal., v. 4, 1967, pp. 390-397.

3. R. E. Barnhill, "Optimal quadratures in $L^{2}\left(E_{\rho}\right)$. II," SIAM J. Numer. Anal. (To appear.)

4. R. E. Barnhill, "Asymptotic properties of minimum norm and optimal quadratures," Numer. Math. (To appear.)

5. R. E. BARNHILL, "An error analysis for numerical multiple integration. I," Math. Comp., v. 22,1968 , pp. $98-109$.

6. S. Bergman, The Kernel Function and Conformal Mapping, Math. Surveys, No. 5, Amer. Math. Soc., Providence, R. I., 1950. MR 12, 402.

7. G. BIRKHOFF \& C. R. DEBOoR, "Piecewise polynomial interpolation and approximation," Approximation of Functions, edited by H. L. Garabedian, Elsevier, Amsterdam, 1965. MR 32 \#6646.

8. P. J. DAvis, "Errors of numerical approximation for analytic functions," J. Rational Mech. Anal., v. 2, 1953, pp. 303-313. MR 14, 907.

9. P. J. DAvis, "Errors of numerical approximation for analytic functions," Survey of Numerical Analysis, edited by J. Todd, McGraw-Hill, New York, 1962. MR 24 \#B1766.

10. P. J. DAvis, Interpolation and Approximation, Blaisdell, New York, 1963. MR 28 \#393.

11. P. J. Davis \& P. Rabinowitz, Numerical Integration, Blaisdell, New York, 1967.

12. M. Golomb \& H. F. Weinberger, "Optimal approximation and error bounds," On Numerical Approximation, edited by R. E. Langer, Univ. of Wisconsin Press, Madison, Wis., 1959. MR $22 \# 12697$.

13. M. Golomb, Lectures on Theory of Approximation, Argonne National Laboratory, Argonne, Ill., 1962.

14. P. C. Hammer \& A. H. Stroud, "Numerical evaluation of multiple integrals. II," MTAC, v. 12,1958 , pp. $272-280$. MR $21 \# 970$.

15. D. SECREST, "Best approximate integration formulas and best error bounds," Math. Comp., v. 19, 1965, pp. 79-83. MR $33 \# 1967$.

16. D. SECREST, "Numerical integration of arbitrarily spaced data and estimation of errors," SIAM J. Numer. Anal., v. 2, 1965, pp. 52-68. MR 31 \#4176.

17. A. H. STroud, "Integration formulas and orthogonal polynomials," SIAM J. Numer. Anal., v. 4, 1967, 381-389.

18. J. L. Synge, The Hypercircle in Mathematical Physics, Cambridge Univ. Press, New York, 1957. MR $20 \# 4073$.

19. R. A. VAlENTIN, "Applications of functional analysis to optimal approximation for analytic functions," Ph. D. Thesis, Division Appl. Math., Brown University, Providence, R. I., 1965 . 